\title{
Relations of Platelet Indices with Endometrial Hyperplasia and Endometrial Cancer
}

\author{
Atilla Karateke ${ }^{1 *}$, Mustafa Kaplanoglu², Ali Baloglu ${ }^{3}$
}

\begin{abstract}
Background: Platelets are blood elements thought to play a role in the immune system and therefore tumor development and metastasis. Platelet activation parameters such as mean platelet volume (MPV), platelet distribution width (PDW), and plateletcrit (PCT) can be easily evaluated with the whole blood count and have been studied as markers of systemic inflammatory responses in various cancer types. Our aim in this study was to evaluate the correlation between endometrial pathologies and MPV, PDW and PCT. Materials and Methods: A total of 194 patients who presented to our clinic with abnormal vaginal bleeding were included in our study. The patients were divided into 3 groups (endometrial hyperplasia, endometrial cancer, control) according to their pathology results. The groups were compared for MPV, PDW, and PCT values obtained from the blood samples taken on endometrial biopsy day. Results: The endometrial cancer patients were the oldest group ( $p=0.04)$. There was no significant difference between the three groups in terms of white blood cell count (WBC), platelet count (PC), and hemoglobin (Hb) level. The highest MPV (p<0.001), PDW ( $p=0.002)$, and PCT ( $<<0.001)$ levels were in the endometrial cancer group, and the lowest levels were in the control group. Conclusions: The easy evaluation of platelet parameters in patients who are suspected of having endometrial pathology is a significant advantage. We found MPV, PDW, and PCT to be correlated with the severity of endometrial pathology with the highest values in endometrial cancer. Studies to be conducted together with different laboratory parameters will further help evaluate the diagnosis and severity of endometrial cancer and precursor lesions.
\end{abstract}

Keywords: Platelet indices - endometrial cancer - endometrial hyperplasia

Asian Pac J Cancer Prev, 16 (12), 4905-4908

\section{Introduction}

Tumor initiation, promotion, progression, metastasis, and clinical findings depend on the response of the host immune system to the tumor cell, as mediated by the inflammatory and coagulation systems (Babu et al., 2014). The modified response of the immune system to cancer development is the result of changes in the inflammatory and coagulation systems. The tumor progresses or is kept under control depending on this response (Mantovani et al., 2008; Hanahan et al., 2011). Although no definite causal connection has been established between inflammation and cancer development in previous evaluations, inflammatory cells and their mediators are thought to play a triggering role in the development of many cancers (Mantovani et al., 2008). We tried to evaluate the prediction accuracy, severity, and prognosis of different cancers by using various inflammatory cell ratios, numbers, and mediators based on this idea. Platelets, neutrophils, lymphocyte parameters, and their ratios with each other have been commonly used in previous studies for similar purposes (Unal et al., 2013; Kemal et al., 2014; Topcu et al., 2014).
Platelets are blood elements that play a role in the coagulation cascade as their main function. However, they can draw various inflammatory cells to the relevant area through their mediators and contribute to angiogenesis. Their tumor cell protective effect against immune system clearance in carcinogenesis in addition to angiogenesis is another identified function (Mantovani et al., 2008). Various platelet markers have been evaluated in various tumors as well as disease and inflammatory pathologies. The platelet count (PC), mean platelet volume (MPV), platelet distribution width (PDW), and plateletcrit (PCT) have been the most commonly used markers to show platelet activity.

Endometrial cancer is usually due to unbalanced estrogen and develops on a background of various degrees of hyperplasia. Inflammatory cells and their changes have been studied in precursor lesions and endometrial cancer, as in many other cancers (Acmaz et al., 2014; Mete et al., 2014). However, differences between platelet parameters in endometrial cancer and hyperplasia and a control group were evaluated only in a limited number of studies. We evaluated the changes in the platelet activity markers of

${ }^{1}$ Hatay Goverment Hospital, Department of Obstetric and Gynecology, Hatay, ${ }^{2}$ Adiyaman University, School of Medicine, Department of Obstetrics and Gynecology Medicine, Adiyaman, ${ }^{3}$ Private Çınarlı Gynecology Hospital, İzmir, Turkey *For correspondence: drkarateke@gmail.com 
Table 1. The demographic data and laboratory findings of patients.

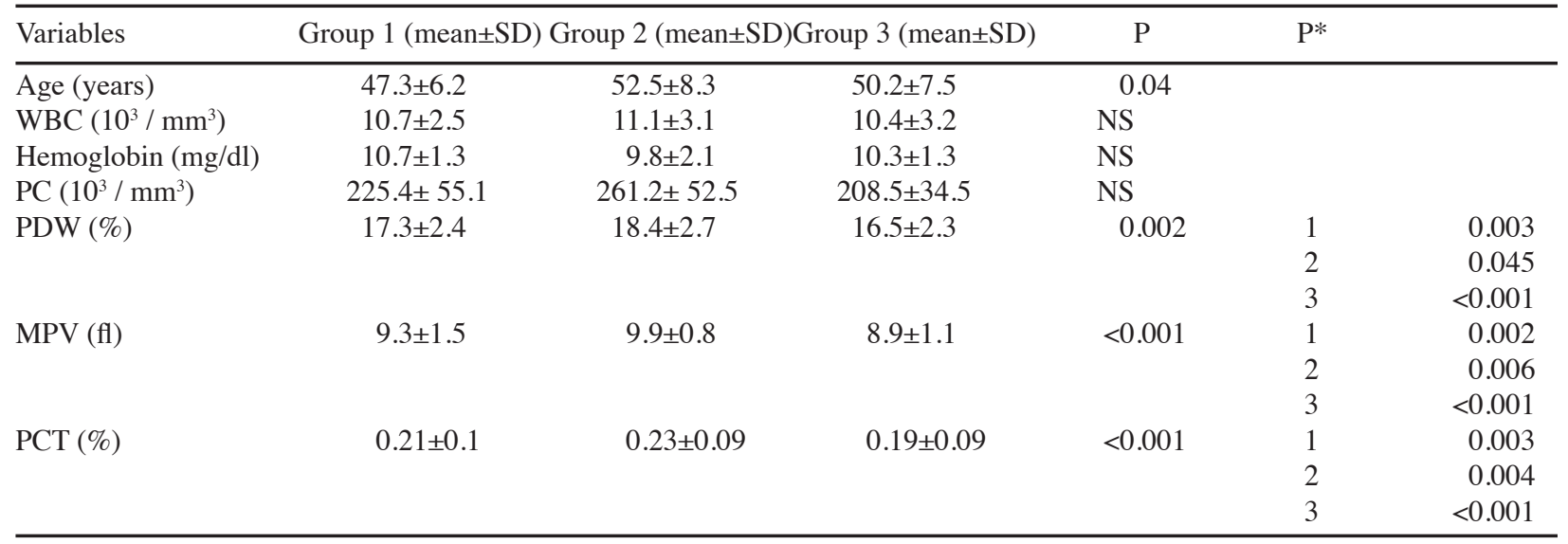

PC, Platelet count; PDW, Platelet distribution width; MPV, Mean platelet volume; PCT, Platelet crit, WBC, White blood cell count, *1, group 1 versus group $2 ; 2$, group 1 versus group $3 ; 3$, group 2 versus group 3

PCT, PDW, and MPV in endometrial hyperplasia and cancer patients in this study.

\section{Materials and Methods}

A retrospective cross-sectional study was conducted at Hatay State Hospital, Department of Obstetric and Gynecology, Hatay, Turkey, between January 2012 and November 2014. The study was approved by the Hatay Goverment Hospital Ethical comitte. A total of 194 cases who presented with abnormal vaginal bleeding and underwent endometrial biopsy were included in this study. The patients were divided into 3 groups according to their endometrial biopsy results, with group I consisting of 55 endometrial hyperplasia cases, group 2 of 34 endometrial cancer cases, and group 3 of 105 normal endometrial biopsy cases.

All patients underwent detailed ultrasonographic and gynecological examinations to eliminate nongynecological reasons for vaginal bleeding. A $10 \mathrm{ml}$ venous blood sample was drawn before any intervention for complete blood count (CBC), human chorionic gonadotrophin, prothrombin time (PT), activated prothrombin time (APTT), and international normalized ratio (INR). Platelets, PCT, PDW, MPV, white blood cell count, and other hematological markers were automatically measured using a Coulter LH 780 Hematology Analyzer (Beckman Coulter Ireland Inc., Mervue, Galway, Ireland). Endometrial curettage was performed after all results were obtained.

Exclusion Criteria: Patients with acute infections, chronic inflammatory disease, malignancies or hematological disorders, those using anti-coagulant treatment, and subjects with a history of hormonal treatment in the last 12 months or blood product administration in the last month were excluded. Patients with endometritis or endometrial polyps were also not included in the study.

\section{Stastical analyze}

Continuous variables are expressed as mean \pm SD. Categorical variables are expressed as percentages. To compare parametric continuous variables, the Student's t test was used; to compare nonparametric continuous variables, the Mann-Whitney U was used. To compare categorical variables, the chi-square test was used. Twotailed $p$ values $<0.05$ were considered to indicate statistical significance. Statistical analyses were performed using SPSS, version 15.0 for Windows.

\section{Results}

The mean ages in the groups were 47.3 $\pm 6.2,52.5 \pm 8.3$ and $50.2 \pm 7.5$ years, respectively. The mean age of patients with endometrial cancer was significantly older than the other two groups $(\mathrm{p}=0.04)$.

Endometrial cancer patients consisted of endometrioid adenocarsinoma $(n=32,94.1 \%)$, mucinous carsinoma $(\mathrm{n}=1,2.9 \%)$ and papillary serous carcinoma $(\mathrm{n}=1$, $2.9 \%$ ). Endometrial hyperplasia cases defined as simple hyperplasia without atypia $(n=45,81.8 \%)$, complex hyperplasia without atypia $(n=5,9.1 \%)$, simple hyperplasia with atypia $(n=3,5.4 \%)$, complex hyperplasia with atypia $(n=2,3.6 \%)$.

The laboratory parameter results are presented in Table 1. There was no significant difference between the groups in terms of WBC ( $>>0.05), \mathrm{Hb}(\mathrm{p}>0.05)$, and PC $(p>0.05)$, while a significant difference was present for PDW ( $\mathrm{p}=0.002)$, MPV $(\mathrm{p}<0.001)$, and PCT $(\mathrm{p}<0.001)$. The highest values for all three parameters were found in the endometrial cancer group and the lowest values in the control group, with a statistically significant difference between the groups.

\section{Discussion}

A CBC is a repeatable, inexpensive, and easily performed laboratory evaluation, and the parameters it includes are frequently affected by cancer and many kinds of inflammatory processes. Platelet parameters (such as PC, MPV, PDW, and PCT) have therefore been used as direct parameters or as cell ratios in patients with precancerous lesions or cancer (Mete et al., 2014).

MPV is the platelet activity marker that is most commonly used to evaluate inflammatory processes and malignancies. Platelets with larger MPV are thought to have granules containing more mediators and thus 
play a bigger role in cancer development and progress (Mantovani et al., 2008). Other important parameters are PDW and PCT. PDW shows the morphological shape changes and reactivity of platelets. Active platelets change shape, undergo spherical transformation, and develop pseudopodia, resulting in changes in platelet size reflected in the laboratory as increased PDW (Leader et al., 2012). The plateletcrit depends on the number of MPV and platelets. It is provided as a direct value in routine whole blood counts and shows what percentage of blood is composed of platelets. It can be considered as analogous to hematocrit and has no clinical meaning by itself (Leader et al., 2012).

Although results vary, the general view is that the number of platelets and active markers and especially the ratio to the number of lymphocytes are correlated with a diagnosis of cancer and the severity of the disorder (Acmaz et al., 2014; Kokcu et al., 2014; Ma et al., 2014; Mete et al., 2014). The number of platelets and neutrophils and the platelet-to-lymphocyte ratio (PLR) have been found to be higher in the healthy control group in studies on precancerous patients and those with endometrial carcinoma. However, it is not possible to obtain definite information on the diagnosis and progression of a patient with endometrial pathology from these results (Acmaz et al., 2014; Mete et al., 2014).

Thrombocyte number and function changes have been identified as part of a paraneoplastic syndrome in many cancers. Several in vivo and in vitro studies have shown that thrombocytes have a proliferative effect on cancer cells and affect their survival through the various mediators they secrete (Anglesio et al., 2011; Cho et al., 2012; Stone et al., 2012). On the other hand, it has been shown that tumor cells are protected from immune clearance in the area where platelets are aggregated (Karpatkin et al., 1981), while metastasis is supported with a selectin-GPII-b dependent mechanism, and tumor adhesion, extravasation, and metastasis are supported via thrombospondin- 1 of the glycoprotein structure secreted from the alpha granules (Tuszynski et al., 1996; Darnik et al., 1997). Accordingly, the number of platelets was found to be higher in cancer patients than the control group in various studies on endometrial and other types of cancer (Acmaz et al., 2014; Kokcu et al., 2014). The main point is the role of active and mediator-secreting platelets in cancer development and progression. It is currently believed in general that the evaluation of platelet activity markers will be valuable for obtaining information on cancer development and progression. We aimed to evaluate platelet parameters in endometrial cancer and hyperplasia in our study. MPV, PDW, and PCT were higher in endometrial cancer patients in parallel to the role considered for activated platelets in cancer development. Our results are also consistent with those on other gynecological and non-gynecological cancers (Anglesio et al., 2011; Acmaz et al., 2014; Kemal et al., 2014; Kokcu et al., 2014). However, data in the literature on the prognostic value of platelet parameters in endometrial cancer are inadequate. We also noticed a change in platelet parameters depending on the severity of the endometrial pathology and believe that this change could be a marker of poor prognosis in endometrial cancer.
We found a significant correlation between platelet indices and endometrial pathology in this study and believe that this will provide useful information on the severity of the disorder before endometrial biopsy as well as the pathology result in a patient suspected to be suffering from an endometrial pathology. The main limitation of our study is the low number of cases. Prospective studies with a large number of cases are required for the evaluation of the correlation between platelet indices and the pathology diagnosis, prediction, and prognosis of cancer patients.

\section{References}

Acmaz G, Aksoy H, Unal D, et al (2014). Are neutrophil/ lymphocyte and platelet/lymphocyte ratios associated with endometrial precancerous and cancerous lesions in patients with abnormal uterine bleeding? Asian Pac J Cancer Prev, $15,1689-92$

Anglesio MS, George J, Kulbe H, et al (2011). IL6-STAT3HIF signaling and therapeutic response to the angiogenesis inhibitor sunitinib in ovarian clear cell cancer. Clin Cancer Res, 17, 2538-48.

Babu SN, Chetal G, Kumar S (2012). Macrophage migration inhibitory factor: a potential marker for cancer diagnosis and therapy. Asian Pac J Cancer Prev, 13, 1737-44.

Cho J, Kennedy DR, Lin L, et al (2012). Protein disulfide isomerase capture during thrombus formation in vivo depends on the presence of $\beta 3$ integrins. Blood, 120, 647-55

Dardik R, Kaufmann Y, Savion N, et al (1997). Platelets mediate tumor cell adhesion to the subendothelium under flow conditions: involvement of platelet GPIIb-IIIa and tumor cell alpha (v) integrins. Int J Cancer, 70, 201-7.

Hanahan D, Weinberg RA (2011). Hallmarks of cancer: the next generation. Cell, 144, 646-74

Karpatkin S, Pearlstein E (1981). Role of platelets in tumor cell metastases. Ann Intern Med, 95, 636-41

Kemal Y, Yucel I, Ekiz K, et al (2014) Elevated serum neutrophil to lymphocyte and platelet to lymphocyte ratios could be useful in lung cancer diagnosis. Asian Pac J Cancer Prev, 15, 2651-4.

Kokcu A, Kurtoglu E, Celik H, et al (2014). May the platelet to lymphocyte ratio be a prognostic factor for epithelial ovarian cancer? Asian Pac J Cancer Prev, 15, 9781-4.

Leader A, Pereg D, Lishner M (2012). Are platelet volume indices of clinical use? A multidisciplinary review. Ann Med, 44, 805-16.

Mantovani A, Allavena P, Sica A, Balkwill F (2008). Cancerrelated inflammation. Nature, $\mathbf{4 5 4}, 436-44$.

Ma X, Wang Y, Sheng H, et al (2014). Prognostic significance of thrombocytosis, platelet parameters and aggregation rates in epithelial ovarian cancer. J Obstet Gynaecol Res, 40, 178-83.

Stone RL, Nick AM, McNeish IA, et al (2012). Paraneoplastic thrombocytosis in ovarian cancer. N Engl J Med, 366, 610-8.

Topcu HO, Guzel AI, Ozer I, et al (2014). Comparison of neutrophil/lymphocyte and platelet/ lymphocyte ratios for predicting malignant potential of suspicious ovarian masses in gynecology practice. Asian Pac J Cancer Prev, 15, 6239-41.

Tuszynski GP, Nicosia RF (1996). The role of thrombospondin-1 in tumor progression and angiogenesis. Bioessays, 18, 71-6.

Unal D, Eroglu C, Kurtul N, et al (2013). Are neutrophil/ lymphocyte and platelet/lymphocyte rates in patients with non-small cell lung cancer associated with treatment response and prognosis? Asian Pac J Cancer Prev, 14, 5237-42.

Ural UM, Sehitoglu I, Tekin YB, Sahin FK (2014). Neutrophil- 
Atilla Karateke and Mustafa Kaplanoglu

to-lymphocyte and platelet-to-lymphocyte ratios in patients with endometrial hyperplasia and endometrial cancer. $J$ Obstet Gynaecol Res, 41, 445-8. 